\title{
Análisis discursivo de los conectores consecutivos aplicado a la versión árabe de la novela Pedro Páramo
}

\author{
Profesor adjunto Dr. Esam Ahmed Nasser \\ Email: esamnasser324@gmail.com \\ Profesora adjunta Hanan Kareem Atta \\ Departamento de Lengua española - Facultad de Lenguas \\ Universidad de Bagdad - Iraq
}

\section{Resumen}

Esta investigación estudia el marcador discursivo de consecuencia tanto en el idioma árabe y el español, por lo que estudiamos los tipos y funciones incluyendo el estudio de contexto representado por la Novela de (Pedro Páramo) del autor (Juan Rulfo) traducido al idioma árabe con nuestra confirmación de ser necesario traducir estos marcadores no sólo dentro de las frases, pero también dentro de los textos a fin de conocer el valor más completo.

Palabras clave: Analisis, marcadores, Pedro Paramo. 


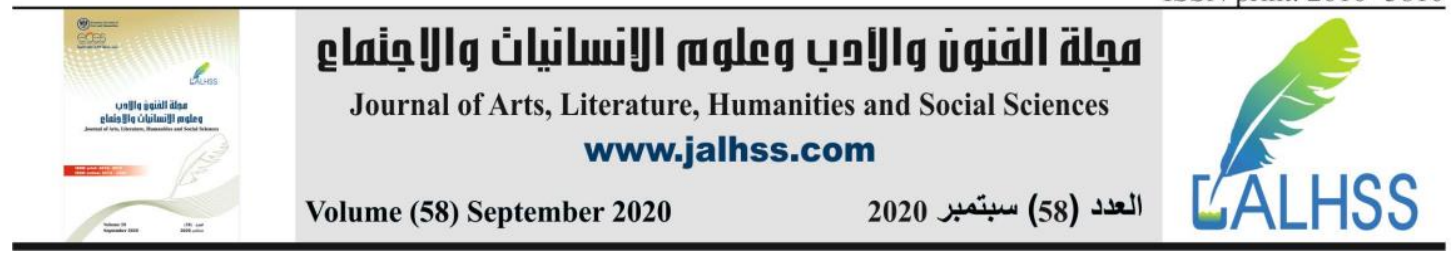

\section{Introducción}

La necesidad de elaborar estudios discursivos y contrastivos, relativos al ámbito de traducción, en las lenguas, aumenta cada día, dicha necesidad resultará imprescindible, si tenemos en cuenta aplicar tales estudios discursivos a una gama variada de obras literarias traducidas a distintas lenguas a fin de conocer a nivel subyacente, la auténtica relación de sus componentes textuales, que ciertamente son los enlaces o los nexos textuales, en su variación, forman parte esencial de dichos componentes.

La idea principal de elaborar esta investigación radica, básicamente, en el gran interés que llevó a los estudios y teorías acerca del análisis del discurso a nivel de las lenguas, pues gracias a esta evolución, podemos comprender los textos de otra forma, más precisa y auténtica, donde podemos profundizarnos más en el nivel semántico interpretativo con la asociación de los otros niveles tanto lingǘsticos como extralingüísticos con el fin de analizar dichos componentes de forma más completa y por lo tanto, se traducen de forma más completa que el estudio elaborado a nivel de la oración.

Nuestro estudio se centra, a nivel discursivo, en investigar sobre los conectores consecutivos a fin de dar a conocer sus funciones y sus valores textuales y si se conservan dichos valores o no a la hora de traducirlos a otra lengua mediante la novela titulada Pedro Paramo del autor Juan Rulfo y su versión en árabe.

\section{Los conectores consecutivos en español}

En este apartado, exponemos un estudio que trata el concepto de los conectores en la lengua española, en particular, los de tipo consecutivo. Antes de dar a conocer, de forma detallada, de los conectores y posteriormente, de su diferencia de los marcadores del discurso, vemos que es pertinente ofrecer informaciones acerca de ¿qué son?.

Los conectores consecutivos son "palabras o grupo de palabras que nos ayudan a conectar palabras, frases u oraciones, gracias a ellas, se pueden expresar relaciones entre distintas ideas y se puede mejorar la expresión al elaborar oraciones más largas y complejas. Existen muchos tipos de conectores en el lenguaje; a demás de los conectores consecutivos. Los más comunes incluyen los conectores de contraste, los de razón y causa, los conectores de propósito, los conectores de edición y los de ejemplificación.

Tomemos los ejemplos siguientes:

1. He estudiado con dedicación, así pues, espero aprobar.

2. He conducido sin descanso durante ocho horas, por lo tanto, nos detendremos en el próximo pueblo.

3. El transporte no ha llegado, de ahí, que todavía estamos aquí.

4. La pista no está disponible, así que no podremos descender. 


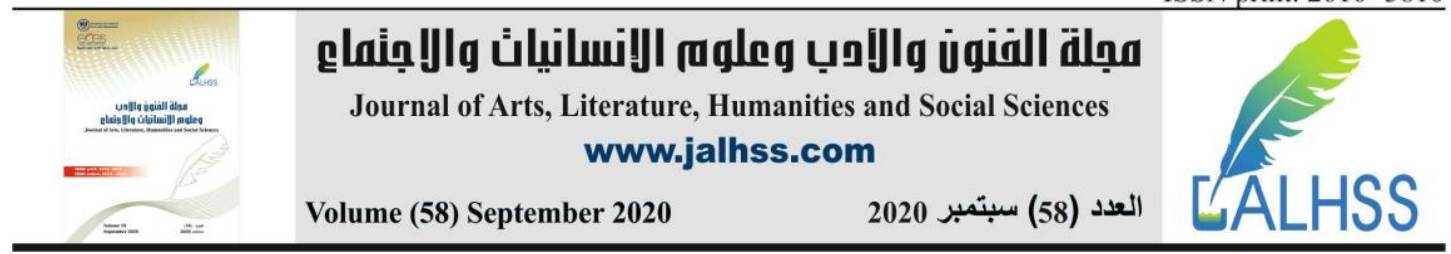

En estos ejemplos, vemos que los conectores consecutivos son los que introducen la preposición subordinada que expresa la consecuencia de lo dicho en la oración, ejercen la conjunción con función consecutiva.

De lo dicho, podemos definir que los conectores consecutivos son como aquellos que unen oraciones en las cuales se une cada oración con otra como lo haré, pues se lo he prometido.

\section{Las características de los conectores consecutivos:}

Los conectores consecutivos se caracterizan por indicar cuál es la conclusión de que se deduce la formación previa a fin de observar su valor, se ha de partir del análisis de las relaciones de causa - consecuencia.

Las oraciones conocidas como "causales" y las tradicionalmente denominadas "consecutivas" coinciden en presentar una relación de causa - consecuencia entre las informaciones conectadas.

\section{Definiciones de conectores causativos - consecutivos}

Dentro de este tipo de conectores, podemos distinguir entre dos grandes grupos, según su función dentro del texto:

En el caso de los conectores de consecuencia, se debe expresar alguna consecuencia o efecto de las ideas que se están planteando u originando en la frase. Pueden ser tanto escritos como orales.

Se llaman "conectores causales", pues se encargan de conectar o juntar frases que están notificando lo que está pasando. En estas palabras, nos dicen las razones o las causas de lo que está ocurriendo.

Existen muchos tipos de conectores de consecuencia en español, que pueden ser utilizados a fin de acompañarse al texto y hacer que las oraciones y frases aparezcan mejor.

Los conectores consecutivos más usados son:

\begin{tabular}{|l|l|}
\hline $\begin{array}{l}\text { Conectores } \\
\text { parenteticos }\end{array}$ & $\begin{array}{l}\text { Conectores } \\
\text { oracionales }\end{array}$ \\
\hline Por lo tanto & $\begin{array}{l}\text { De manera } \\
\text { que }\end{array}$ \\
\hline $\begin{array}{l}\text { Por } \\
\text { consiguinte }\end{array}$ & Asi que \\
\hline Por ende & De ahi que \\
\hline Pues & $\begin{array}{l}\text { De modo } \\
\text { que }\end{array}$ \\
\hline A causa de & \\
\hline Por eso & \\
\hline Asi & \\
\hline Asi pues & \\
\hline
\end{tabular}




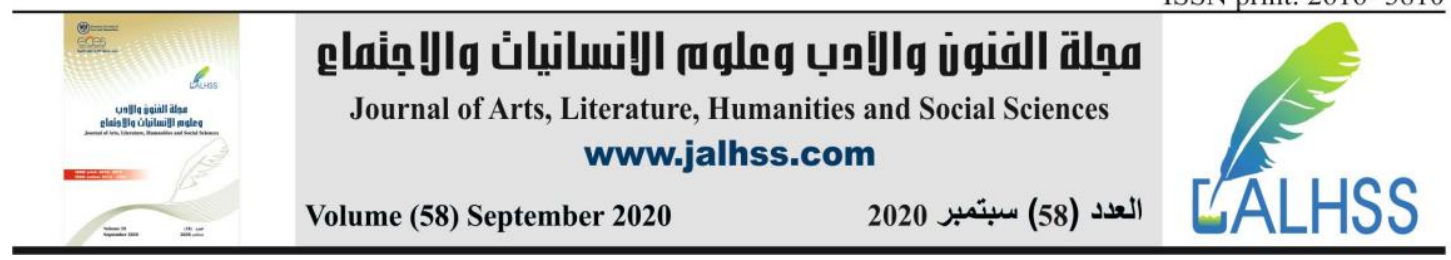

\section{Los conectores consecutivos correspondientes al efecto o consecuencia}

Proponen establecer un límite a lo que ya se expresó o a la parte inicial del enunciado; por otro lado, pueden expresar una dificultad o una oposición a que no impide el cumplimiento de lo dicho en la oración principal.

\section{Una visión general a los conectores:}

Los conectores se usan en el desarrollo de los textos regularmente, por otro lado, marcan el curso u orientan la forma en la que el fragmento se interpreta con la relación con el contexto o con las inferencias obtenidas de él, de igual forma, estas palabras proponen al lector el sentido al texto, enlazan diferentes maneras de los enunciados que lo componen.

Un conector puede ser una palabra, una oración o conjuntos de elementos, por lo tanto, unen lo más breve con lo más extenso.

\section{Los marcadores consecutivos en español desde el marco del discurso}

A nuestro juicio, vemos que es muy necesario aquí resaltar los valores discursivos de los conectores consecutivos en el español y en el árabe, dichos valores son más allá que los lingüísticos y gracias a ellos, podemos demarcar la noción más completa y precisa de estos conectores. Domínguez García (Domínguez García, 1997, pag.34), habla del concepto de la "categoría funcional" o mejor dicho, del "espacio funcional", que consiste en " la idea de tratar a un macro funcional que engloba una serie de valores cuyos límites son imprecisos".

Cabe aclarar la cuestión terminológica existente entre "conectores" o" marcadores". En este sentido, vemos que hay dos opiniones principales a cerca de esta cuestión terminológica, la primera opinión se inclina hacia la indiferencia funcional en la operación de dichos conectores o marcadores, la cuestión no va más que terminológica, debido a que en Europa es preferido llamarse a dichas conjunciones como "Conectores", a distinción de los Estados Unidos, donde los denominan "Marcadores", dicha opinión es apoyada por (Pons Bordería (1998: 22), sin que se dé mucha cuenta de la diferencia funcional y discursiva.

La segunda opinión se centra en el funcionamiento de dichos conectores o marcadores, partiendo de la idea de considerar el término "Marcador" como hiperónimo del término "Conector", es decir, este es un tipo de aquello.

En España, podemos encontrar las dos opiniones juntas, pues según Cuenca (Cuenca, 2006: 29), el término "Marcador" es más preciso que el de "Conector", pues es adoptado más a los estudios del discurso y según las funciones discursivas que realiza dentro del texto o del habla, funciones que no se limitan a conectar entre las oraciones, sino mantienen una relación extralingüística también (como la relación pragmática) y a través de esta relación textual y contextual en que los marcadores del discurso mantienen un papel principal en manejar su idea principal. Podemos saber la auténtica función de dichos marcadores y por consiguiente, su término pertinente.

Por último, cabe dar a conocer que la definición más propicia y conocida a cerca de los marcadores del discurso que hemos encontrado es la de José Portelés, en su libro 


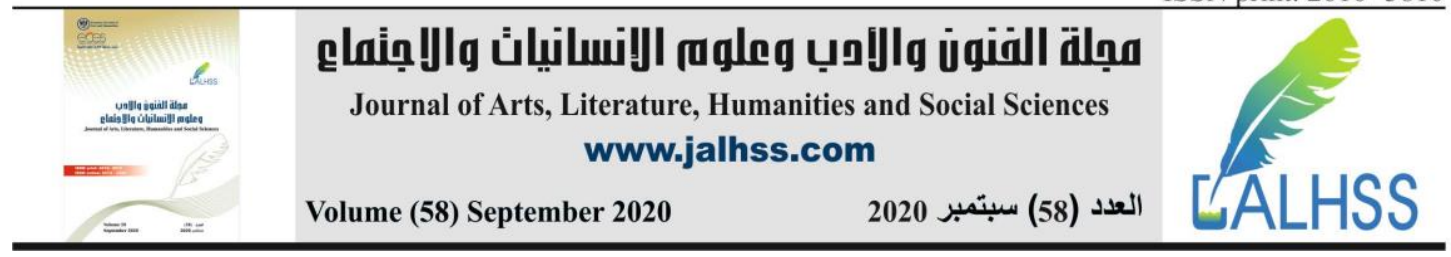

Marcadores del Discurso: Un estudio discursivo e interdisciplinario, vemos que son "unidades lingüísticas invariables, no ejercen una función sintáctica en el marco de la predicación oracional y poseen un cometido coincidente en el discurso: el de guiar, de acuerdo con sus distintas propiedades morfosintácticas, semánticas y pragmáticas, las inferencias que se realizan en la comunicación" (Lázaro, José portolés, Marcadores del discurso, 1998). .

\section{Los conectores consecutivos en árabe desde el marco de la lingüística}

En la lengua árabe, igualmente que en la lengua española, encontramos la existencia de varias partículas o conectores de consecuencia, pues cada partícula o conector cuenta con usos concretos. Existe también, en esta lengua, la posibilidad de tener una partícula o conector más que una finalidad de uso. Antes de profundizarnos en los detalles de uso de estas partículas, es pertinente explicar la noción de la consecuencia en el idioma árabe y en este sentido, cabe hablar del concepto de conjunción o conexión en la lengua árabe.

En el texto árabe, podemos decir que los estudios lingüísticos y discursivos han tenido evolución en cuanto a lo lingüístico, igual que el texto español. En cuanto a la estructura lingüística, que forma las partículas conjuntivas o se llaman también conectores conjuntivos de tipo causal, en este caso, se usan:

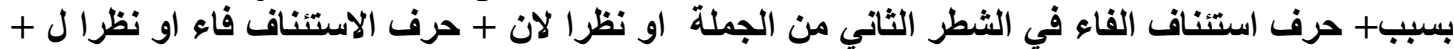
حرف الاستئناف الفاء (د. محمود عبد الله جفال الحديد أدوات الربطو الوصلئل الوصل في اللغة العربية، ص 21-23).

Ejemplos:

1. (Las áreas de las tierras agrícolas de Egipto han aumentado a causa de, por ende, por consiguiente de la construcción de la alta presa).(

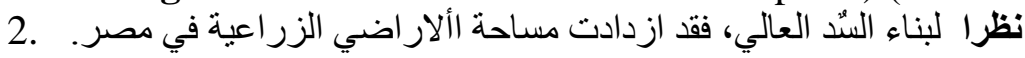

A causa de la construcción de la alta presa, han aumentado las áreas agrícolas en Egipto.

Podemos decir que existen dos tipos de estructuras en cuanto al árabe, la primera estructura viene con valor de causalidad y la segunda es de valor consecutivo. Ambos tipos requieren a dos partes de oración, requieren a un motivo a fin de completar su significado, pues es lógico que se vincule la primera parte con la segunda con una partícula o un conector pertinente. Hay muchos tipos de partículas o conectores.

En cuanto a las formas lingüísticas con las partículas de valor consecutivo, vemos que esta forma se compone, en su mayoría de dos partes, igual que las formas anteriores. La forma con valor consecutivo, que se compone de primera parte, contiene "una predicación más que la partícula de consecuencia más la segunda parte que incluye el valor consecutivo que es un resultado lógico de esta predicación".

Estos conectores normalmente vinculan a palabras, frases o párrafos. Pueden ser nombres, verbos o adverbios, sean simples o compuestos. En relación a su función, hacen la función de relacionarse, de hacer comentarios. 


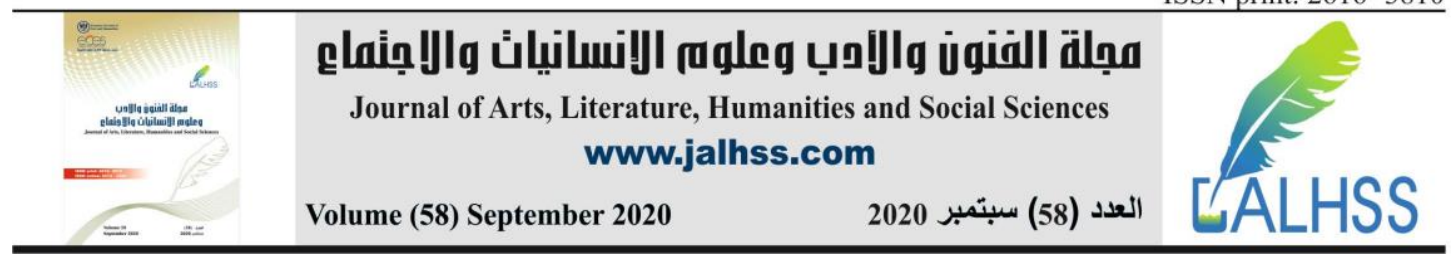

Si decimos:

لم يكن للاتر الك مدنية وحضارة قديمة، بل كانوا اشبه بالبدو، ولهذا (ولذلك) اطلق عليهم الجاحظ اعراب -

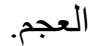

A los turcos, no tenían ni ciudad ni civilización antigua, sino eran como beduinos, por lo tanto (por eso), El Ȳahiid

les nombró como beduinos aljamiados.

Vemos que el tipo de la oración es consecutivo.

Las partículas que acompañan a las oraciones consecutivas son varias como:

و وعلى هذا، ونتيجة لهذا، ولهذا، ومن هنا -

لقد فثل في تجاربه وخسر كل شيء، ولهذا فقد قرر ان يهاجر من بلده. -

El fracasó en sus pruebas y perdió todo, por lo tanto, (por consiguiente), decidió marcharse de su país.

En árabe, podemos dividir los conectores en dos tipos, "Conectores sintácticos", los cuales son acompañantes fonéticos singulares, que relevan el significado contextual entre las partes de una oración o entre las oraciones, delimitando el modo de las oraciones. Estos conectores son imprescindibles, en su función, a la oración u oraciones.

El segundo tipo de estos conectores son "Conectores textuales", mediante los cuales, conectan a las partes del discurso y no hace falta que revele el estado fonético, sino que resulta suficiente vincular a las partes del discurso enunciado, incluso que el hablante puede ampliar su enunciación.

\section{Los marcadores en árabe desde la disciplina discursiva}

Al igual que el estudio español arriba mencionado a cerca del uso más amplio de los marcadores a nivel del discurso, comprendido esto como texto o habla. En atabe, los marcadores discursivos, en general, y los consecutivos, en particular, juegan un papel tan importante en los textos desde dos dimensiones principales, que son:

- La primera dimensión consiste en la necesidad de entender los marcadores del discurso, no solamente desde el mero valor lingüístico, sino desde su valor discursivo, sea oral o escrito; es decir, actualmente y de acuerdo con los estudios de la ciencia del texto y del habla, dichos marcadores ya no son únicamente como conectores que enlazan entre dos o más oraciones y mantienen cohesión entre sí, sino son como marcadores que juegan el papel más importante, en el discurso, que los otros componentes, en formar la coherencia general del texto o del habla y en este caso, su valor discursivo es distinto entre un discurso y otro. Este valor discursivo depende del contexto, pues, estos marcadores se convierten de meros conectores con su valor léxico a otro con valor contextual que diferencia de un texto a otro.

- La segunda dimensión necesaria en relación con lo expuesto en el punto (1) consiste en la necesidad de entender el valor discursivo de los marcadores discursivos según el contexto. Este entendimiento necesita decir que las relaciones que manejan cualquier discurso, este manejo de relaciones es hecho por los marcadores discursivos a fin de entender su contenido de forma lógica. En este sentido, podemos asegurar que el valor 


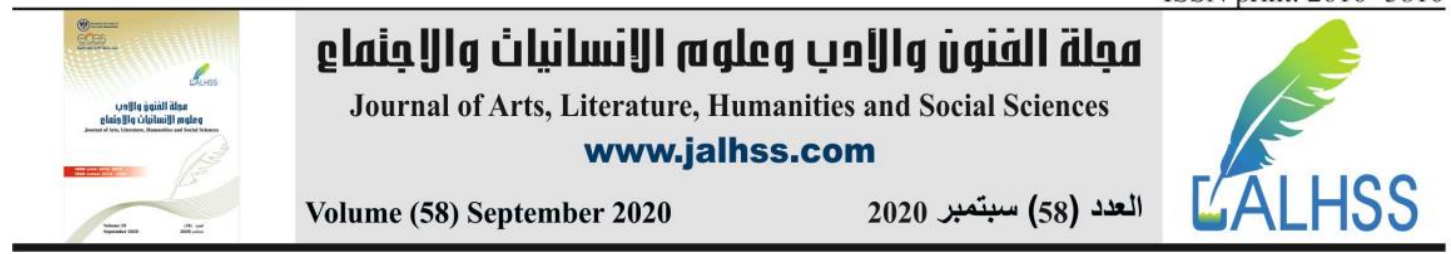

oracional de estos marcadores no es suficiente a fin de conceder las referencias interpretativas completas dentro del discurso, sino, requiere a unas situaciones dentro del discurso a adaptarse al nivel de la unidad discursiva y a tener la noción referencial e interpretativa completa. Tomemos unos ejemplos en el árabe:

\section{Ahmed durmió y el camaleón cambió de color de piel نام احمد وغيرت)}

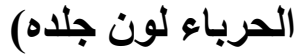

En esta oración, vemos que el significado o el valor referencial de esta oración no es nítido, aunque los componentes lingüísticos son bien colocados, pero si ponemos esta oración dentro de una escena textual, podremos encontrar que su valor semántico y pragmático es muy diferente, pues si decimos:

$$
\text { وأبصر أحمد وغيرت الحرباء لون جلدها، بعد أن كسر فرع الشجرة التي نام عليها أحمد، فقزع أحمد لما استيقظ }
$$

(Ahmed durmió y el camaleón cambió de color de piel tras haber roto la rama de árbol sobre la cual durmió y al haberse despertado, se asustó de verlo).

De este ejemplo, deducimos que la diferencia semántica - pragmática de tipo interpretativo se queda más clara que en el ejemplo anterior, pues se entiende muy bien que Ahmed rompió la rama de árbol sobre la cual había dormido y al haberse despertado, tuvo susto de ver al camaleón. Este valor semántico - pragmático de tipo interpretativo puede ser dentro del contexto. Existe también situaciones en que dichos valores pueden proceder desde fuera del contexto como en:

$$
\text { نام أحمد وغيرت الحرباء لون جلاها، بعد أن كسر فرع الثجرة التي نام عليها أحمد، ففزع أحمد لما استيقظ }
$$$$
\text { وأبصر تلك الحرباء أنرياء }
$$

(Dijo sonriente: Ahmed durmió y el camaleón cambió de color de piel tras haber roto la rama de árbol sobre la cual dirimió y al haberse despertado, se asustó de verlo). En este ejemplo, es necesario descubrir nociones que son exteriores al contexto, pues alguien puede preguntar por este contexto, la respuesta puede ser que " Ahmed se pasó por un zoo y al mismo tiempo, vio a un camaleón que cambió de color de piel. Esta historia puede ocurrir en leyendas, por ejemplo.

\section{Estudio analítico de los marcadores discursivos de consecuencia en la novela Pedro Paramo y su versión en árabe}

En este apartado, nuestro objetivo es elegir unos ejemplos tomados de la novela Pedro Paramo del autor Juan Rulfo, nacido el día 16 de mayo de 1917 en Jalisco. Es novelista, cuentista, fotógrafo y editor, a Rulfo se le reconoce, sobre todo, por su volumen de cuentos El llano en llamas (1953) y su primera novela Pedro Páramo (1955). A partir de la aparición de estos títulos mantuvo un contacto frecuente con el cine; su segunda novela, El gallo de oro (1958), el cortometraje El despojo (1959) y su participación en el filme La fórmula secreta (1964) son producto de ello. La novela de Pedro Paramo, nacido en Hums en (صالح علماني), ha sido traducida dos veces, una vez por el traductor sirio llamado por Șaleḩ AlmanI 1949 y murió en Madrid en 2019. Fue traductor palestino especialista en traducir libros y novelas del español al árabe. El segundo traductor es مروان ابر اهيم صديق (Marwan Ibrahim Șediq) . 


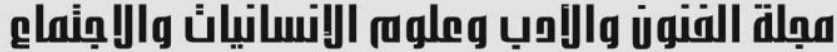 \\ Journal of Arts, Literature, Humanities and Social Sciences \\ www.jalhss.com \\ Volume (58) September 2020 \\ العدد (58) سبتمبر 2020}

Es traductor de varias novelas al lado de esta novela, pues es traductor al árabe de la novela (El túnel) y la de (Señora de Rojo sobre Fondo Gris). He aquí algunos ejemplos de dicha novela con sus análisis discursivos relativos a los marcadores discursivos de tipo consecutivo:

\begin{tabular}{|l|l|l|l|l|}
\hline Número & Texto original & Texto terminal & Texto \\
terminal 2
\end{tabular}




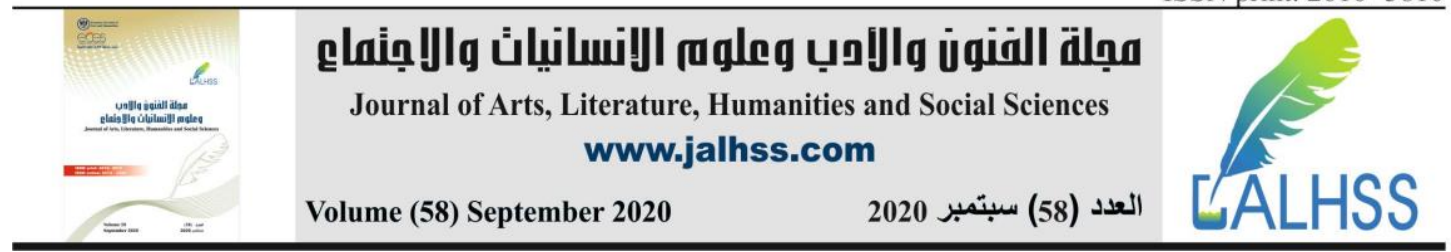

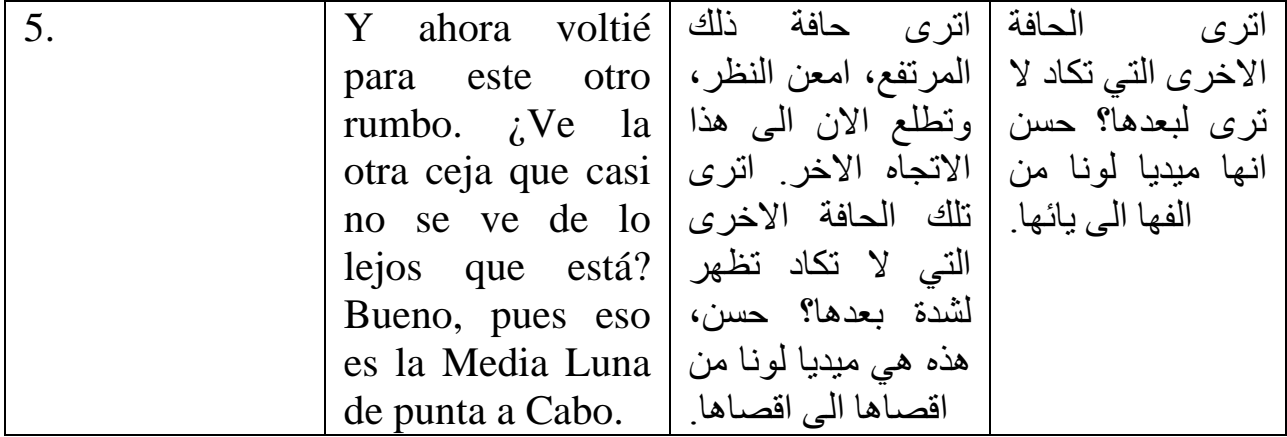

Empecemos nuestro estudio analítico con el esquema siguiente:

En este esquema, hemos elegido unos ejemplos tomados de la novela Pedro Paramo relativos al uso de los marcadores del discurso de tipo consecutivo y podemos señalar a través de las dos traducciones elaboradas en el árabe a dicha novela.

He aquí la explicación de nuestras notas producidas en las dos traducciones elaboradas.

1. Las notas que podemos registrarnos en estos ejemplos seleccionados y de acuerdo con el orden cronológico mencionado en el esquema, son las siguientes:

1. En el ejemplo primero, vemos que los dos traductores no han elegido una forma equivalente a nivel discursivo), mientras que, a nosotros, podemos decir que una propuesta de (هكذ) del marcador discursivo de tipo consecutivo

Y como unatraduccion adecuada y precisa, a nivel discursivo, al marcador consecutive así seráal.

2. En el ejemplo Segundo, vemos que el marcador discursivo de consecuencia pero se ha traducido de forma impropia, en este caso, podemos decir que aunque todos sabemos que la traducción لكن discursiva al árabe, sin embargo, al leer el ejemplo mencionado y ver las dos traducciones de este marcador, árabe del marcador pero (لكن) podemos decir que no tenemos dos partes de consecuencia de tipo contradictorio que implican el uso de este marcador discursivo y traducirlo de forma literal, sino que es una descripción al estado del retrato y de esta forma, por lo cual, vemos que una traducción muy conveniente al árabe de este marcador y según el discurso será

$$
\text { اذ انها الصورة الوحيذة او وهي الصورة الوحياة. }
$$

3. En el ejemplo tercero, vemos que ambos traductores mencionados han traducido Mi madre siempre fue enemiga de retratarse. Decía que los retratos eran cosa de brujería. Y así parecía ser; porque el suyo estaba lleno de lo ha traducido por agujeros como de aguja, vemos que el primer traductor y el segundo traductor lo ha traducido como

فو الدتي كانت دائما علوة لالتقاط الصور. وكانت تقول ان الصور امر من امور السحر، وييلو انها كنلك فعلا، لان صورتها كانت مليئة بثقوب صنيزة من الثئقوب الابرة. 


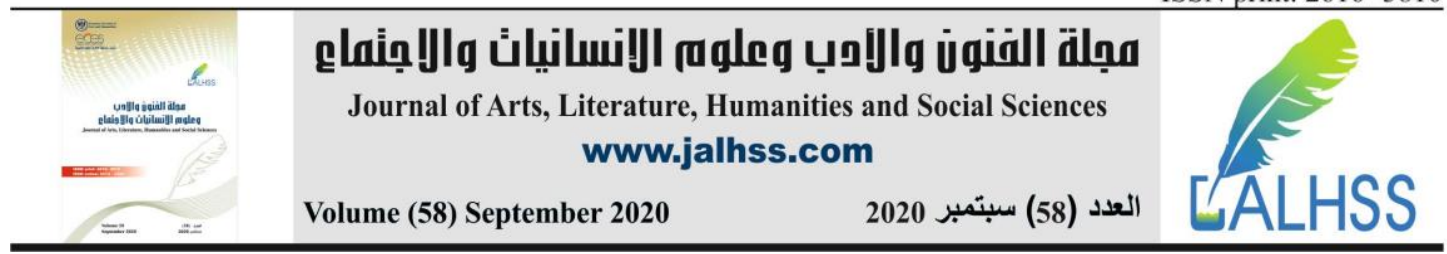

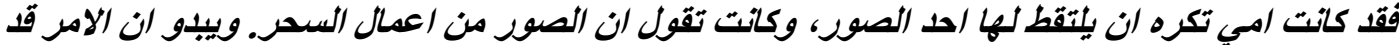
تم بهذا الشكل لان الصورة كانت مليئة بالثقوب كما لو انها ثقوب البرة الأبرة
}

ويبيدو انها كذلك فعلا

En ambas traducciones, vemos que (así parecía ser) se ha traducido por el primer traductor como una

y en la segunda como

$$
\text { ويبيدو ان الامرقد تم بهذا الشكل. }
$$

En este ejemplo, podemos decir que ni la primera traducción ni la segunda en que pudieron los ambos traductores dar equivalencia discursiva en árabe a este marcador discursivo preciso del marcador así es

$$
\text { و هكذا هياو وتبدو هي هكذا او وتبدو الصور هكذا. }
$$

Nuestras razones de elegir estas formas como equivalentes a este marcador discursivo radican en que (parecer y ser) son dos verbos auxiliares de un mismo significado en árabe; asimismo, el uso del verbo parecer en imperfecto tiene valor de presente, pues cuya acción sigue incluso en el futuro.

4. En el ejemplo cuarto, vemos que ambos traductores mencionados han traducido-Mire usted —me dice el arriero, deteniéndose-: ¿Ve aquella loma que parece vejiga de puercos? Pues detrasito de ella está la Media luna, vemos que el traductor (صالح علماني) lo ha traducido como

$$
\text { (انظر ، اترى تللك الر ابية التي تبدو مثل مثانة خنزير؟ ور اءها تماما توجد ميديا لونا). }
$$

y el segundo traductor (مروان ابر اهيم) lo ha traducido como

$$
\text { ويبدو انها كذلك فعلا. الربوة التي تبدو مثل مثانة الخنزير؟، فخلفها مباشرة تقع (ميديالونا). }
$$

En ambas traducciones, vemos que una vez el marcador del discurso de tipo "consecutivo" no se ha traducido por el primer traductor por ninguna equivalencia discursiva y otra vez en la lengua arabe que sirve a "consecuencia", pero aqui tenemos un valor distinto de (الفاء) traducido por "consecuencia", el cual es el valor ilativo, puesto que viene este marcador tras una pregunta.,

la traducción correcta, en este caso, es حسنا.

5. En el último ejemplo, el cual es ( Y ahora voltié para este otro rumbo. ¿Ve la otra ceja que casi no se ve de lo lejos que está? Bueno, pues eso es la Media Luna de punta punta a Cabo ).

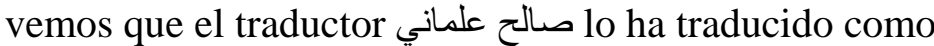

$$
\begin{aligned}
& \text { (اترى حافة ذلك المرتفع، امعن النظر، وتطلع ان الى هذا الاتجاه الاخر. اترى تلك الحافة الحافة الاخرى التي لا }
\end{aligned}
$$

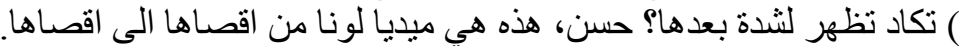

y el segundo traductor (مروان ابر اهيم) lo ha traducido como

$$
\text { (اترى الحافة الاخرى التي تكاد لا ترى لبعدها؟ حسن انها ميديا لونائها من الفها الى يائها.). }
$$




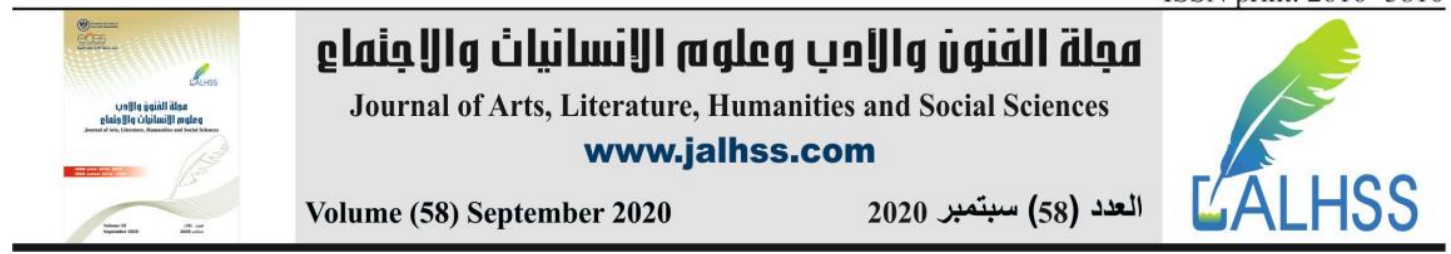

En ambas traducciones, vemos que el marcador del discurso de consecuencia pues Se ha traducido por حسن, mientras que este marcador es precedido por el adverbio bueno, que significa también حسن. Vemos que en ambas traducciones, la equivalencia discursiva a este marcador a base de su valor discursivo será اذا

\section{Conclusiones finales}

Tras el fin de elaboración de este trabajo de investigación acerca del uso de los marcadores discursivos de tipo consecutivo en las ambas lenguas, lengua árabe y española y desde una perspectiva analítica moderna, vemos, a nivel de conclusión, lo siguiente:

1. Es muy necesario, actualmente, informarse de los valores discursivos de tipo consecutivo y entender sus componentes, tanto lingüísticos como extralingüísticos, centrándose en los componentes extralingüísticos (discursivos), pues estos son los que nos importan más a la hora de marcar sus valores dentro del discurso, sea oral o escrito, y comprender su situación contextual.

2. Este proceso de marcación de los valores discursivos se pone más complicado al ampliar el estudio hacia lo contrastivo entre ambas lenguas, en este caso, hablamos de lengua árabe y española, a través de la traducción, pues tratamos con dos estructuras lingüísticas y discursivas distintas, cosa que requiere centrarnos en los aspectos extralingüísticos que mantienen relación con la función de dichos marcadores dentro del texto a fin de concretar el auténtico valor discursivo y después, transmitirlo a la otra lengua de forma precisa.

3. A la hora de aplicar el estudio analítico al lenguaje literario y su relación con la traducción, es muy necesario atenderse a las características propias de este lenguaje y a su papel en nuestra elección de este o de aquel valor discursivo.

\section{Agradecimiento}

Nos dirigimos, con palabras de gratitud, a todas las personas, sean familiares, parientes, gente de amistad, quienes han participado, de una forma $\mathrm{u}$ otra, en brindarnos la oportunidad de elaborar esta investigación. 


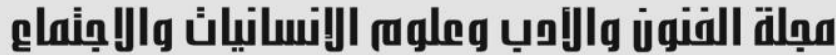 \\ Journal of Arts, Literature, Humanities and Social Sciences www.jalhss.com

\section{Bibliografia}

1. Domínguez García, Naomi: Conectores: Estado de la cuestión, Universidad de Salamanca, 1997.

2. Pons Borderim Salbador: Conexión y conectores. Estudio de su relación en el registro informal de la lengua, Cuadernos de Filología, Anexo XXVII, Valencia, Universitat de València, 1998. |

3. Lazaro, José Portelés: Marcadores del Discurso, Ariel. Barcelona, 1998

4. Cuenca, Josep Maria: La connexió $i$ els connectors. Perspectiva oracional $i$ textual, Vic, Eumo, 2006.

\section{Bibliografía en árabe}

$$
\text { 1. د. محمود عبد الله جفال الحديد مذكرات في أدوات الربطو الوصل في اللغة العربية،2004. }
$$

